\title{
Laboreal
}

Volume $5 \mathrm{~N}^{\circ} 1$ | 2009

Ergonomia e desenvolvimento sustentável

\section{Resiliência e fragilidade dos sistemas de trabalho e sustentabilidade : estudos de casos de sistemas sócio-técnicos complexos no Brasil na área nuclear, aviação e emergência}

Resiliencia y fragilidad de los sistemas de trabajo y sustentabilidad: estudios de caso de sistemas socio-técnicos complejos en Brasil en la área nuclear, aviación y emergencia

Résilience et fragilité des systèmes de travail et développement durable : études de cas de systèmes socio-techniques complexes au Brésil dans les domaines du nucléaire, de l'aviation et de l'urgence Resilience and brittleness of work systems and sustainability : Brazilian case studies in nuclear, aviation, and emergency domains

José O. Gomes, Paulo V. R. Carvalho, David D. Woods, Tahar Hakim Benchekroun e Marcos R. S. Borges

\section{(2) OpenEdition}

\section{Edição electrónica}

URL: http://journals.openedition.org/laboreal/10605

DOI: $10.4000 /$ laboreal. 10605

ISSN: 1646-5237

\section{Editora}

Universidade do Porto

Refêrencia eletrónica

José O. Gomes, Paulo V. R. Carvalho, David D. Woods, Tahar Hakim Benchekroun e Marcos R. S.

Borges, «Resiliência e fragilidade dos sistemas de trabalho e sustentabilidade : estudos de casos de sistemas sócio-técnicos complexos no Brasil na área nuclear, aviação e emergência », Laboreal [Online], Volume $5 \mathrm{~N}^{0} 1$ | 2009, posto online no dia 01 julho 2009, consultado o 10 outubro 2019. URL http://journals.openedition.org/laboreal/10605; DOI : 10.4000/laboreal.10605

Este documento foi criado de forma automática no dia 10 outubro 2019.

Laboreal está licenciado com uma Licença Creative Commons - Atribuição-NãoComercial 4.0 Internacional. 


\section{Resiliência e fragilidade dos sistemas de trabalho e sustentabilidade : estudos de casos de sistemas sócio-técnicos complexos no Brasil na área nuclear, aviação e emergência}

Resiliencia y fragilidad de los sistemas de trabajo y sustentabilidad: estudios de caso de sistemas socio-técnicos complejos en Brasil en la área nuclear, aviación y emergencia

Résilience et fragilité des systèmes de travail et développement durable : études de cas de systèmes socio-techniques complexes au Brésil dans les domaines du nucléaire, de l'aviation et de l'urgence

Resilience and brittleness of work systems and sustainability : Brazilian case studies in nuclear, aviation, and emergency domains

José O. Gomes, Paulo V. R. Carvalho, David D. Woods, Tahar Hakim Benchekroun e Marcos R. S. Borges

\section{NOTA DO EDITOR}

Manuscrito recebido em : Fevereiro/2009

Aceite após peritagem em : Junho/2009 


\section{Introdução}

1 Uma condição básica para que uma organização alcance alta confiabilidade e resiliência é superar as tendências reativas, construindo antecipações aos problemas e a eventos inesperados e não desejados. Tal organização deve ser capaz de observar o passado com clareza, produzindo compreensão e reflexão sobre os micro-incidentes, as restrições ao trabalho dos operadores e as estratégias desenvolvidas pelas pessoas de forma a aprender e prevenir a ocorrência de falhas nas organizações (Woods, 2005). A Engenharia de Resiliência tem como propósito permitir às pessoas e às organizações a se tornarem atentas e sensíveis aos modelos de riscos que adotam de forma a controlar a origem e os caminhos das falhas (Dekker, 2006).

2 Os acidentes em sistemas sócio-técnicos (Challenger, Columbia, colisão aérea da Gol/ Legacy, queda da aeronave Black Hawks dos EUA no Iraque, etc.) têm mostrado como diversas organizações, todas com múltiplas camadas de defesas e sistema de controle, não conseguiram balancear os riscos da segurança com a pressão produtiva. Esses acidentes evidenciaram padrões clássicos de deriva das organizações em direção a uma operação mais eficiente e menos segura, produzindo acidentes. Alguns desses padrões são : enfatizar mais a produção que a segurança, considerando os êxitos do passado como razão de confiança no futuro; processos fragmentados de resolução de problemas ; falta de reavaliação das estimativas quando novas evidências se acumulam ; perturbações/interrupções dos fluxos de informação entre departamentos da organização dificultando a comunicação, resultando em organizações cegas e incapazes de aprender com os incidentes de menor importância (CAIB, 2003; Snook, 2000 ; Carvalho, Gomes, Huber \& Vidal, 2009).

3 Se considerarmos que os padrões descritos acima são quase os mesmos para todo um conjunto de acidentes maiores em sistemas sócio-técnicos complexos, então, para poder evitar tais acidentes, necessita-se criar a antecipação (foresight), monitorando o nível de risco do sistema através de seu ciclo vital completo e identificando os sacrifícios na tomada de decisões (sacrifice decisions), isto é, as compensações da segurança/produção são feitas pelas pessoas todos os dias. Além disso, precisamos entender como se obtém êxito frente às restrições à atividade de trabalho (e se esse êxito poderia conduzir a maiores falhas) e como as pessoas aprendem e se adaptam para garantir a segurança em um mundo pleno de lacunas, perigos e conflitos de metas e objetivos (Hollnagel \& Woods, 2005 ; Adamski \& Westrum, 2003; Cook, Render \& Woods,2000).

Durante o seu trabalho diário, as pessoas atuam em diversos papéis dentro de uma organização complexa com várias camadas de barreiras de defesa em profundidade. Neste cenário complexo, as pessoas não estão totalmente conscientes dos potenciais caminhos de falhas que podem emergir conforme elas desenvolvem as estratégias locais para face frente às restrições e à complexidade do sistema. A multiplicidade de tarefas para manter uma operação eficiente e sem falhas e a conseqüente sobrecarga cognitiva, normalmente impede às pessoas de refletir sobre o resultado de suas ações e aprender com elas. Uma organização resiliente deve proporcionar meios para superar esta situação, monitorando, entendendo, refletindo e aprendendo a partir dessas estratégias, identificando ameaças e riscos à segurança. Falhar em aplicar esses princípios leva a organização a atuar em um modo reativo (e hindsight), numa condição de luta constante contra o perigo (Woods, 2005). 
Os estudos de caso apresentados neste artigo procuram aplicar os conceitos e métodos de engenharia resiliência, bem como relacioná-los com o conceito de sustentabilidade dos sistemas sócio-técnicos complexos, num país de desenvolvimento industrial recente que é o caso do Brasil. Os casos abordam os domínios nuclear, aviação e emergência. Em uma Usina de Energia Nuclear, foram analisados micro incidentes durante a operação da planta. As análises mostram as ações de controle dos operadores usadas para solucionar pequenos conflitos que se apresentaram no nível operacional, e como eles organizaram os recursos requeridos para sua ação/cognição, ou seja, o material, a parte social, e características culturais do ambiente. $\mathrm{O}$ quadro (framework) de micro incidente permite uma visão antecipada das ações de controle dos operadores, permitindo a análise sistêmica e o pensamento crítico sobre a possibilidade, de que essas situações problemáticas relativamente pequenas nas camadas fracamente acopladas do sistema, possam conduzir a resultados negativos em algum momento no futuro (Carvalho, Santos, Gomes \& Borges, 2008).

6 Também foram estudados os conflitos entre metas e objetivos no sistema de transporte por helicópteros para as plataformas de petróleo na Bacia de Campos no Brasil, para descobrir o quão resiliente e frágil é o sistema de transporte por helicóptero, dada as demandas de produção e pressões econômicas vigentes. A análise permitiu conhecer conflitos entre meta e objetivos que se apresentaram nas fronteiras do funcionamento entre as diversas organizações envolvidas e como as pessoas, atuando em seus diversos papéis, adaptam-se a esses conflitos, e as implicações destas adaptações para segurança e resiliência do sistema como um todo (Gomes et al., 2009).

O terceiro estudo caso relacionado à pesquisa tem por objetivo analisar a simulação da resposta a uma emergência nuclear sob uma abordagem da ergonomia cognitiva e engenharia de resiliência. Esta simulação acontece anualmente como forma de treinamento para responder eficazmente a situações e eventos ligados às emergências nucleares. Ela se desenvolve na cidade de Angra dos Reis, em cujo município está localizado o parque nuclear brasileiro de produção de energia e congrega 26 organizações privadas e públicas, nos níveis federal, estadual e municipal (Costa et al., 2008).

8 Por fim, acreditamos que a resiliência dos sistemas sócio-técnicos complexos é uma condição, entre outras, para manter a sustentabilidade das organizações. Para isto, o uso dos conceitos, da metodologia e das ferramentas que fazem parte da Análise Ergonômica do Trabalho (AET), é uma condição sine qua non para compreender as organizações identificando resiliência e fragilidades que interferem no funcionamento dos sistemas produtivos complexos. O conceito de desenvolvimento sustentável dos sistemas de trabalho empregado neste artigo se baseia, em parte, na resiliência do mesmo, tanto ao nível de projeto quanto de funcionamento. Portanto, desenvolver a resiliência, compreendendo o nível de projeto e operação, é uma condição necessária e fundamental. Por resiliência de projeto, compreendemos a atividade desenvolvida na concepção dos sistemas produtivos complexos cujo processo permite em suas várias etapas realizar atividades de simulação que permitam, por exemplo, visualizar as atividades futuras de funcionamentos, identificando gaps e bugs na relação entre tecnologia, pessoas e organizações (Adamski \& Westrum, 2003). E por resiliência de funcionamento ou operação compreendemos a capacidade continua e ininterrupta do sistema em adaptar-se à variabilidade de situações e sempre se antecipar às situações não desejáveis para garantir uma confiabilidade e eficiência permanentes. 


\section{Metodologia}

9 A Engenharia de Resiliência proporciona uma estrutura metodológica e a Análise de Tarefas Cognitivas (CTA - Cognitive Task Analysis), as técnicas para analisar o trabalho em sistemas complexos, utilizadas nos diversos estudos de casos descritos artigo: micro-incidentes em sala de controle de uma usina nuclear, sistema de transporte por helicópteros e a simulação de resposta à emergência nuclear. A CTA é um nome geral que engloba um conjunto de métodos e técnicas usados para compreender e descrever os aspectos cognitivos das atividades diárias de trabalho, incluindo como os profissionais vêem o trabalho que fazem, e como eles dão sentido aos eventos e restrições que encontram durante o desempenho de suas atividades (Crandall, Klein \& Hoffman, 2006). Esses métodos dependem de um acesso direto aos profissionais ou especialistas ou trabalhadores experientes em domínios específicos dos quais se busca extrair informações.

10 De acordo com a abordagem da engenharia de resiliência, para permitir que as pessoas e organizações possam tomar melhores decisões no tradeoff produção versus segurança num contexto dinâmico e competitivo, não é suficiente a organização possuir apenas um sistema de gestão de riscos, com barreias de segurança e uma engenharia voltada para a proteção contra eventos adversos. Em organizações resilientes, a segurança deve ser fazer parte das tomadas de decisão diárias, por meio de uma revisão ativa dos modelos de risco e avaliação da efetividade das ações corretivas Uma organização segura precisa ser dinâmica, engajada, informada e informativa para ser capaz de manter um balanço de produção versus segurança adequado em um longo período de tempo (Woods, 2005). Desta maneira, uma organização para se tornar resiliente precisa desenvolver maneiras de gerar informações sobre como a organização está realimente operando e por que as pessoas estão operando desta maneira.

11 Assim, em vez de se focar em como o trabalho deve ser feito (as regras prescritas e tarefas), nós nos voltamos em compreender como e porque o trabalho está sendo feito de uma forma particular, considerando as restrições ou limites que conformam o trabalho, e analisando os modelos de risco que as pessoas estão usando durante suas decisões de sacrifício. Esta abordagem identifica a variabilidade das atividades dos trabalhadores e como suas opções - o quê, quando e como agir - são permitidas e/ou ajudadas ou restringidas pelo ambiente de trabalho. Em meio a estas condições, os trabalhadores podem gerar uma grande variedade de padrões de trabalho, incluindo comportamentos desconhecidos e inovações nas práticas de trabalho que precisam ser monitoradas para identificar as implicações âmbito da relação produção versus segurança. Como há diferentes tipos de restrições que podem moldar o comportamento dos trabalhadores, várias dimensões de análise são necessárias, como mostradas na figura 1. 
Figura 1 : As diversas dimensões da análise

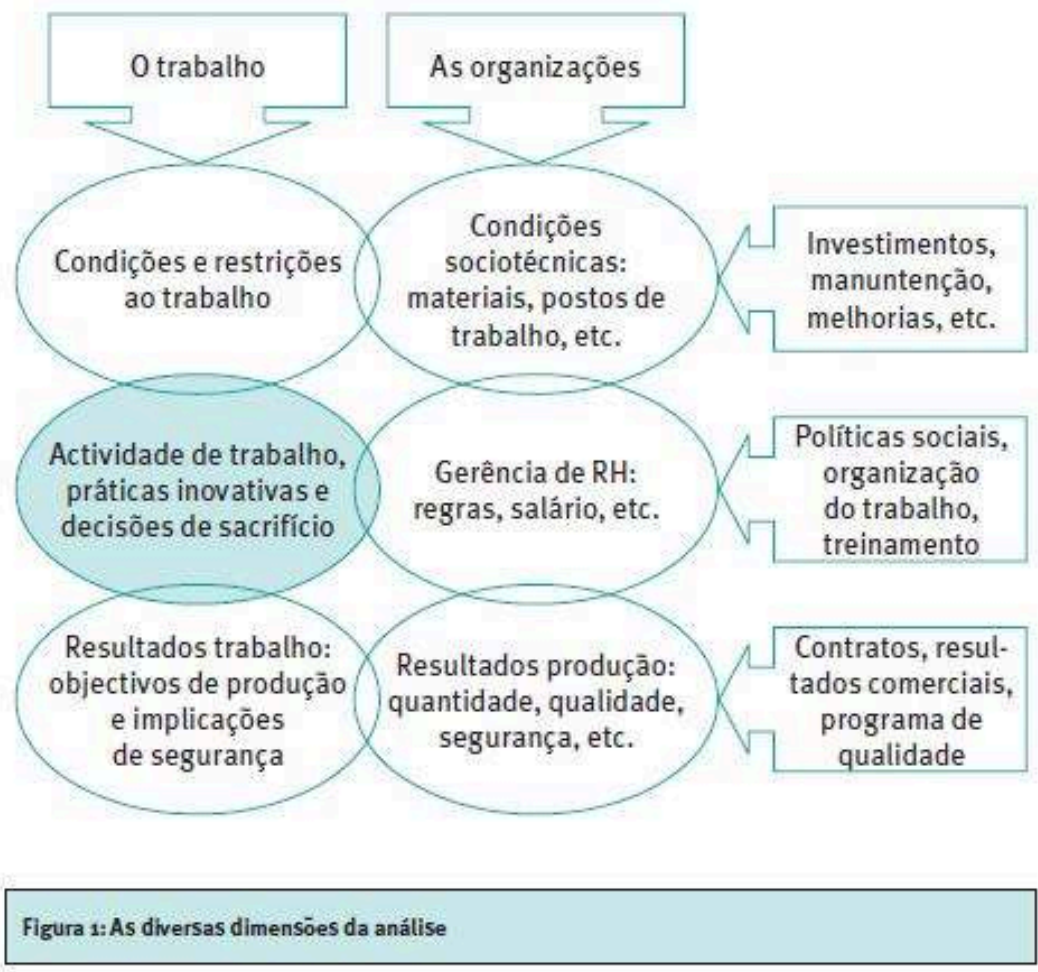

12 Nos casos que serão apresentados a seguir, diversos métodos e técnicas da Análise de Tarefas/Atividades Cognitivas foram usados tanto na fase de coleta quanto de análise e apresentação dos dados, conforme as características de cada ambiente de trabalho e os objetivos específicos de cada um dos estudos.

\section{Análise dos Casos : nuclear, aviação e emergência}

\subsection{Tomada de decisões operacionais em usinas nucleares}

13 Neste estudo o objetivo foi observar como os operadores de sala de controle de usinas nucleares lidam com situações novas, ou pelo menos não esperadas, as quais definimos como situações de micro incidentes (Carvalho et al., 2008 ; Carvalho, Vidal \& Carvalho, 2007 ; Carvalho, Santos \& Vidal, 2006) de modo a avaliar como os operadores tomam decisões (tradeoffs segurança versus produção) e usam o suporte dos procedimentos operacionais.

14 A atividade de trabalho dos operadores foi gravada mediante o uso de equipamentos de áudio e vídeo. Conforme a abordagem de estudos em situação real de trabalho da ergonomia, os operadores receberam só uma instrução: comportar-se de forma tão normal quanto possível, apesar dos equipamentos de gravação e da presença dos analistas na sala de controle. Quatro ergonomistas observaram o trabalho dos operadores na sala de controle e coletaram os dados para o estudo. O procedimento consistiu de 3 fases : 1) Coleta de dados, 2) Preparação dos dados e 3) Análise. Para compreendermos as decisões dos operadores ao lidar com micro-incidentes, o conteúdo das gravações de áudio e vídeo do trabalho dos operadores foi codificado em diversas categorias conforme a tabela 1. 
Tabela 1 : Esquema de codificação

\begin{tabular}{|c|c|}
\hline Categoria & Definiçāo \\
\hline Ponto de ruptura - Decisão & $\begin{array}{l}\text { Ponto de ruptura da operaçao "normal" motivando decisao que gera } \\
\text { um curso de açao (CuA) diferente do previsto no procedimento. Por } \\
\text { exemplo, usar um atalho, parar um processo, esperar para ver como o } \\
\text { micro incidente evolui, enviar um operador ao campo etc. }\end{array}$ \\
\hline Entrada & $\begin{array}{l}\text { Informaçao que conduz a uma avaliaçáo alterada que requer uma } \\
\text { decisao. Identificaçào de quando o topico relativo a decisao foi } \\
\text { introduzido e que fatores novos causaram a mudança. }\end{array}$ \\
\hline Instigado por & Quem identificou a necessidade de tentar resolver um problema. \\
\hline Envolvidos & $\begin{array}{l}\text { Pessoal ervolvido desde a identificaçào do problema/ auxilio na } \\
\text { resoluçáo até a tomada de decisáo. }\end{array}$ \\
\hline Meta & $\begin{array}{l}0 \text { objetivo da decisao. Verbalmente declarado ou deduzido pelo } \\
\text { investigador. Metas incluem parar um processo, partir um sistema, } \\
\text { realizar testes etc. }\end{array}$ \\
\hline Razào & $\begin{array}{l}\text { Baseada na meta. Por exemplo, a meta pode ser a parada de um } \\
\text { processo, a razăo era minimizar o dano potencial da evoluçăo do } \\
\text { micro incidente. Pode ser declarado mas, freqüentemente, tem que } \\
\text { ser deduzido. }\end{array}$ \\
\hline Opçoes e consequiências & $\begin{array}{l}\text { Opçðes disponiveis como meios altemativos de solucionar o proble- } \\
\text { ma identificado. Dentre essas opç̋es podem estar năo fazer nada ou } \\
\text { esperar. As conseqüências se referem ao que aconteceria se estas } \\
\text { opçoes fossem selecionadas em vez do CuA escolhido. Mais umavez, } \\
\text { opçoes e consequências podem ser declaradas, mas algumas vezes } \\
\text { precisam ser deduzidas. }\end{array}$ \\
\hline Tempo & $\begin{array}{l}\text { O tempo decorrido desde quando o problema foi identificado ate a } \\
\text { tomada de decisáo. }\end{array}$ \\
\hline
\end{tabular}

15 Seguindo o método proposto 15 micro incidentes (Mis) foram analisados, sendo 10 durante a parada de usina, 4 durante a partida e 1 durante o treinamento em simulador. Dentre estes MIs observamos que procedimentos foram seguidos em apenas 3 ocasiões, sendo que em uma delas durante o treinamento em simulador, seguir o procedimento a risca sem considerar o estado geral da planta foi o que engendrou o micro incidente, conforme observação do próprio instrutor do simulador. Em diversas ocasiões, operadores, mais especificamente Supervisores de Turno, tiveram que arbitrar situações onde procedimentos geravam conflitos, como nos casos de liberação/ suspensão de testes nos quais os procedimentos de teste eram incompatíveis com a programação dos testes : a condição operacional da usina prevista no procedimento de teste não era compatível com o momento planejado para sua realização. Nestes casos o Supervisor tentou por duas vezes realizar os testes (contrariando um dos procedimentos) e teve de suspendê-los em função de dificuldades operacionais.

Resumimos a seguir os principais resultados do estudo :

- Os operadores de sala de controle resolvem problemas gerados por micro incidentes e tomam suas decisões basicamente a partir de reconhecimento de padrões e de regras condição-ação implícitas. Estas regras parecem ser derivadas muito mais da experiência e treinamento (conhecimento tácito) do que dos procedimentos operacionais padrão.

- Os problemas são resolvidos em série à medida que vão emergindo, quando há alguma mudança observável no estado do sistema. Há pouca evidência de geração e comparação de opções.

- As restrições do ambiente (técnicas, sociais, culturais) limitam severamente a possibilidade de antecipação e a quantidade de opções disponíveis. 

contam os operadores das usinas para realizar as tarefas de operação. Entretanto, os resultados do estudo apontam problemas em relação ao modo de utilização, elaboração e modificação dos procedimentos. Enquanto a estrutura legal adota como um valor seguir procedimentos a risca - estabelecendo punições em caso de não cumprimento de procedimentos - as restrições técnicas, organizacionais e culturais vão de encontro a este objetivo. que se supõe sejam seguidas à risca chamada por Perin (2005) de "cultura do controle", possui limitações em função da dificuldade dos projetistas de preverem todas as ações que serão efetivamente necessárias em situações novas e devido às restrições impostas pelo contexto de trabalho. treinamento em simulador. Este procedimento manda reduzir a pressão até 80 bar e não considera inteiramente as variabilidades do processo, gerando dúvidas inclusive quanto a pertinência da instrução e forma de utilização. Quando questionados pelo instrutor o motivo pelo qual estariam seguindo uma instrução às cegas, o que estaria agravando o acidente simulado, os operadores alegaram questões estruturais/legais, como a obrigatoriedade de seguir os procedimentos à risca.

Diversos micro incidentes analisados mostraram situações onde os operadores, em função da situação, das metas e objetivos, das pressões, de seu estado físico e emocional, e também em face de dificuldades inerentes às postulações e incongruências contidas nos próprios procedimentos, lançam mão de estratégias cognitivas baseadas em regras condição-ação implícitas, tentativa e erro, analogias para tomar decisões e flexibilizar procedimentos. Estas condições obrigam os operadores a realizar modificações ad hoc em procedimentos (não cumprimento de requisitos escritos) durante a operação, apesar da evidente ansiedade que este tipo decisão provoca em função da cultura de controle da organização, como no exemplo dos micro incidentes relacionados a liberação/suspensão de testes. Micro incidentes durantes os testes emergiram da relação entre a necessidade do Supervisor seguir o planejamento das tarefas (pressão da produção) com requisitos contidos nos procedimentos de testes (realizar testes com o reator no estado sub crítico frio, que não era o estado do reator no momento planejado para os testes), Assim, por duas vezes o Supervisor toma a decisão de não seguir o requisito do procedimento de teste (reator sub crítico frio) e libera os testes conforme o plano de tarefas, os quais tiveram que ser suspensos após pressões do demais operadores em função de problemas no processo de resfriamento da planta. 0 uso de estratégias baseadas em analogias ("subir a potência em $5 \%$ para ver se pára a oscilação"), sem o suporte necessário para saber se a estratégia poderia afetar a segurança, permitem indicar que tipo de modelo local de risco vem sendo adotado por cada tipo de operador (supervisores, operadores da planta).

Por outro lado, é importante observar que o modelo local de risco é reforçado pelos sucessos obtidos nestas flexibilizações trazendo como conseqüência natural uma aumento da confiança neste tipo de estratégia, que tende a ser cada mais utilizada, mas que necessariamente demandas novas formas de abordar este contexto fundamental para a segurança dos processos, tal como apontada pela engenharia de resiliência.

Laboreal, Volume $5 \mathrm{~N}^{\circ} 1$ | 2009 


\subsection{Sistema de Transporte por Helicópteros na Bacia de Campos}

Esta pesquisa, baseada na análise da atividade cognitiva dos pilotos que voam na Bacia de Campos a serviço da Petrobrás, busca identificar os fatores contribuintes e os contrantes que interferem na atividade dos pilotos, cujas consequências afetam o desempenho operacional do sistema de transporte como um todo e, consequentemente, a segurança de vôo. Contrantes são fatores que de alguma maneira dificultam o que é feito e, principalmente, a forma prescrita de como as coisas deveriam ser feitas, sendo então determinantes da criação de diversas estratégias adaptativas por parte dos agentes. Como regra geral, estas estratégias adaptativas podem tanto facilitar o trabalho das pessoas quanto contribuir para ocorrências não desejadas no sistema. Normalmente, elas são insuficientes, de modo isolado, para provocar incidentes ou acidentes e, portanto, permanecem invisíveis nas análises mais tradicionais, baseadas em relatórios de perigo, análise de acidentes, inspeções de reguladores etc. (Carvalho et al., 2006). Entretanto, a carga de trabalho agregada, ou o desgaste gerado pela acumulação de diversos "pequenos" desvios nos modos de trabalho sob as pressões de um ambiente organizacional que visa maior produção com menor custo, pode se constituir num solo fértil para a emergência de grandes perdas, impactando na resiliência e segurança do sistema (Woods, 2005 ; Woods, 2006).

23 Métodos baseados em entrevistas foram utilizados, devido as diversas dificuldades que a observação direta do trabalho dos pilotos traria ao projeto de pesquisa. Os sujeitos da pesquisa (pilotos e co-pilotos de helicóptero) foram sistematicamente entrevistados no aeroporto quando não estavam voando. As entrevistas foram gravadas com o consentimento dos entrevistados e posteriormente transcritas para a realização da análise. A pesquisa foi coordenada por dois ergonomistas e contou o auxílio de 5 estudantes de graduação na sua realização. Além disso, todo o processo contou com a participação de especialistas em segurança de vôo e aviação offshore. Os resultados finais foram validados junto aos pilotos e co-pilotos das diversas companhias.

Como conseqüência da estrutura do sistema de gestão, cada ator persegue seus próprios objetivos, em função das suas áreas de responsabilidade. Conflitos de interesses crescem, em particular, no caso dos pilotos de helicóptero que devem reportar problemas de manutenção, os quais podem prejudicar seus próprios ganhos e a rentabilidade da empresa, pois o tempo de inspeção e paradas para manutenção diminuem o tempo efetivamente voada pelo qual ambos, pilotos e empresas, são remunerados (a estrutura contratual atualmente em vigor no transporte offshore no Brasil privilegia as horas voadas pelas aeronaves e/ou o número de aeronaves que estão disponíveis para vôo). Caso um helicóptero seja retirado para manutenção, a empresa não gera receitas e reduz a capacidade de fornecer o serviço estipulado no contrato. Além disso, relatórios oficiais de problemas técnicos podem deixar fora de serviço a aeronave até a próxima inspeção da Agência Nacional de Aviação Civil (ANAC) (realizada pelo contratante) que ocorre a cada 15 dias. Como resultado, o helicóptero pode ficar fora do serviço por mais tempo até ser realizada a inspeção para liberar o equipamento para serviço.

Assim, as relações organizacionais e financeiras criam pressão para manter os helicópteros voando. Sob essa pressão, pilotos encaram um dilema para decidir se um problema técnico é suficiente ou não para iniciar um ciclo oficial de manutenção. Os pilotos até reconhecem indícios de problemas (vibração, ruído etc.), que seriam 
indicadores prematuros de possíveis falhas no equipamento, e poderiam reportar à manutenção antes que estes problemas se tornem uma ameaça à segurança de vôo. Entretanto, enviar o helicóptero para a manutenção retira-o de serviço, perdendo-se tempo de vôo que contraria um calendário exigente e uma demanda sempre crescente pelo serviço, reduzindo as receitas de pilotos e empresas.

Esta situação é um exemplo de decisão de sacrifício alicerçada na pressão de ser mais rápido, melhor e mais barato (faster, better, and cheaper). A descoberta desse dilema no transporte offshore na Bacia de Campos permitiu à equipe investigar como o sistema foi adaptado a lidar formalmente e informalmente com estas pressões. A figura 2 utiliza uma estrutura de fluxo para capturar a decisão de sacrifício dos pilotos. 0 sistema adapta e caracteriza os problemas em duas classes : aqueles severos o suficiente para requererem o processo oficial, incluindo a espera da inspeção após o reparo já ter sido feito, e outras que são leves o bastante (de acordo com a experiência do piloto) para serem reportadas diretamente (mas informalmente) ao funcionário de manutenção ou de investigação.

o dilema enfrentado pelos pilotos refere-se a reportar oficialmente uma condição ou não. A segunda opção permite manter a aeronave em serviço (minimizando perdas financeiras relativas ao tempo de vôo), enquanto a manutenção avalia a informação (gravidade da situação), ou encomenda peças. A manutenção pode até manter o helicóptero em terra. A decisão de reportar oficialmente ou não é sempre do piloto. Entretanto, as pressões contratuais polarizam o processo decisório dos pilotos que é agravado pela dificuldade técnica que os pilotos podem ter (em função de sua experiência, formação tempo de vôo etc.) para interpretar e avaliar a gravidade dos problemas técnicos.

Este espaço de manobra surge, em parte, porque há uma diferença entre dois conjuntos de regras. As regras regulatórias são mais estritas do que aquelas da Relação de Equipamentos Mínimos (REM), que é uma lista de itens específicos sem os quais uma aeronaves não pode voar, regulado por normas internacionais. Portanto, apesar de existirem problemas, reportá-los pode não ser obrigatório e, se eles ficam aquém das regras mais rígidas, podem ser reportados diretamente para a área de manutenção, sem passar pelo processo oficial de relatório.

29 A análise permitiu identificar que a decisão de sacrifício dos pilotos depende, por uma lado da capacidade do piloto para perceber de sintomas fracos de problemas durante as diversas fases de um vôo, e por outro lado ter a expertise necessária para, a partir dos sintomas percebidos, discriminar situações de manutenção adiáveis daquelas críticas e inadiáveis.

Outro ponto relevante refere-se a relações comerciais da companhia de helicópteros com os seus pilotos e com o principal cliente que é a companhia de petróleo. Acreditamos que um entendimento mais global do contexto pode permitir agir na melhoria da segurança como um todo. Um sistema de segurança proativo deve ser capaz de, através de indicadores, emitir sinais relativos aos pontos frágeis do sistema, antecipando-se aos eventos adversos, através de uma monitoração contínua e constante. Isto pode ser possível a partir do conhecimento e da interpolação entre aspectos locais/situados e os aspectos organizacionais deste sistema complexo, que envolve várias organizações dispersas espacialmente e temporalmente, cujas sincronizações de objetivos e metas revelam-se frágeis em determinados contextos e resilientes em outros. 
Figura 2 : Diagrama de fluxo do processo de tomada de decisão dos pilotos

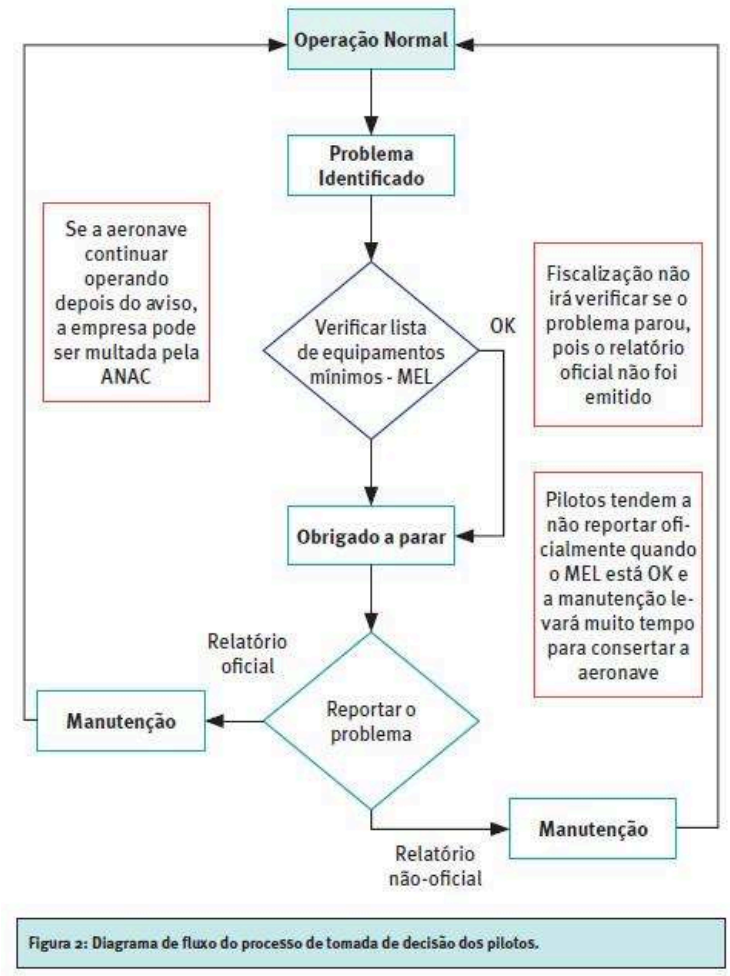

\subsection{Analise Ergonômica da Simulação da Resposta à Emergência Nuclear}

Este trabalho apresenta resultados de uma análise do trabalho cognitivo (ATC) de uma simulação de acidente nuclear. Registros audiovisuais foram coletados de uma equipe de sala de emergência composta por indivíduos de 26 agências diferentes no momento em que respondiam a múltiplos cenários de um acidente nuclear simulado. Esta simulação fez parte de uma atividade nacional de treinamento de resposta a emergências em uma usina nuclear. Múltiplas técnicas de ATC para coleta, análise e representação dos dados (Crandall et al., 2006) foram usadas de modo a obtermos um melhor entendimento das dimensões cognitivas da atividade e identificar padrões de coordenação de equipe e gestão de crises surgidos no treinamento simulado. Este estudo foi coordenado por dois ergonomistas e contou com a participação de alunos de graduação e pós-graduação.

As atividades das pessoas na sala envolvidas na simulação Plano de Emergência Externo (PEE) foram representadas ao longo da linha temporal, apresentada na figura 3. Na representação, para cada minuto da simulação existe uma linha com diversos campos a serem preenchidos. Por exemplo, entre 9:45 e 9:46 da simulação, atividades envolvendo Perguntas, Pedido de Silêncio e Chegada de pessoas foram observadas. Os campos correspondentes a cada uma dessas subcategorias e ao horário 9:45 seriam, então, marcados.

Para representar as diversas atividades das equipes, foram criadas categorias de ações. Categorias comuns foram rotuladas para expressar momentos chave da simulação, 
assim como, para simplificar a representação para análise. Essas categorias estão listadas na tabela 2 .

Tabela 2 : Categorias de ações e descrições

\begin{tabular}{|c|c|c|}
\hline Categorias & Acçōes & Descriçāo \\
\hline \multirow{6}{*}{ Comunicação } & Conversa em grupos pequenos & Pessoas falando em grupos pequenos \\
\hline & $\begin{array}{l}\text { Mensagem da planta/nova } \\
\text { informaçăo }\end{array}$ & Novos eventos, cenários, informaç̧̄es \\
\hline & $\begin{array}{l}\text { Explicaçăo de detalhes/conhe- } \\
\text { cimento especifico }\end{array}$ & $\begin{array}{l}\text { Explicaç̧es técnicas para dividir } \\
\text { conhecimento }\end{array}$ \\
\hline & Comunicaçào externa (celular) & $\begin{array}{l}\text { Comunicaçào com o exterior da sala } \\
\text { de emergencia }\end{array}$ \\
\hline & $\begin{array}{l}\text { Repetiçào de informaçōes } \\
\text { conhecidas }\end{array}$ & $\begin{array}{l}\text { Resumos para manter } 0 \\
\text { ambiente em acordo }\end{array}$ \\
\hline & Pergunta & Dóvidas dos participantes \\
\hline \multirow{4}{*}{$\begin{array}{c}\text { Ordem/ } \\
\text { Comando }\end{array}$} & $\begin{array}{l}\text { Comando para os membros } \\
\text { da equipe }\end{array}$ & $\begin{array}{l}\text { Ordens dadas pelo coodenador ou } \\
\text { outro lider }\end{array}$ \\
\hline & Aprovaçào e tomada de decisåo & $\begin{array}{c}\text { Decisôes relativas ao que ser feito } \\
\text { em cada caso }\end{array}$ \\
\hline & Pedido de Silêncio & Evitar a dispersao do grupo \\
\hline & Pedido de Informaçao & Evitar informaçðes confusas \\
\hline \multirow{2}{*}{$\begin{array}{c}\text { Físico/ } \\
\text { Tecnologia }\end{array}$} & Problemas tecnologicos & $\begin{array}{l}\text { Recursos tecnologicos } \\
\text { inadequados }\end{array}$ \\
\hline & Chegada de pessoas & $\begin{array}{l}\text { Substituiç̧os, almoço, } \\
\text { chegada de pessoas }\end{array}$ \\
\hline
\end{tabular}
participante da simulação. Enquanto deveriam existir 26 cores na linha do tempo completa, devido às limitações do tempo de gravação, apenas as ações dos indivíduos que aparecem no vídeo foram representadas na linha do tempo. A figura 3 mostra um exemplo da estrutura da linha do tempo de $10: 30$ até $11: 00$.

eito de análise e compreensão, comentários sobre algumas ações específicas e de grande relevância foram feitos nas caixas que possuem uma marca vermelha à direita, no topo. Foram alocados números para cada seqüência de ações que possuíam a mesma origem, i.e. uma nova mensagem chega para a equipe. Essa nova mensagem corresponde à serie 24 na sequência de ações observadas. Então a série 24 será escrita na caixa dessa ação. Em seguida a essa mensagem, irão ocorrer ações envolvendo explicação de detalhes, perguntas e tomada de decisão. Cada uma dessas ações, nessa mesma sequência, pertencerá a serie 24 . Quando um novo evento ocorrer, a série 25 será dada à primeira ação desse evento. 
Figura 3 : Visão detalhada da linha do tempo

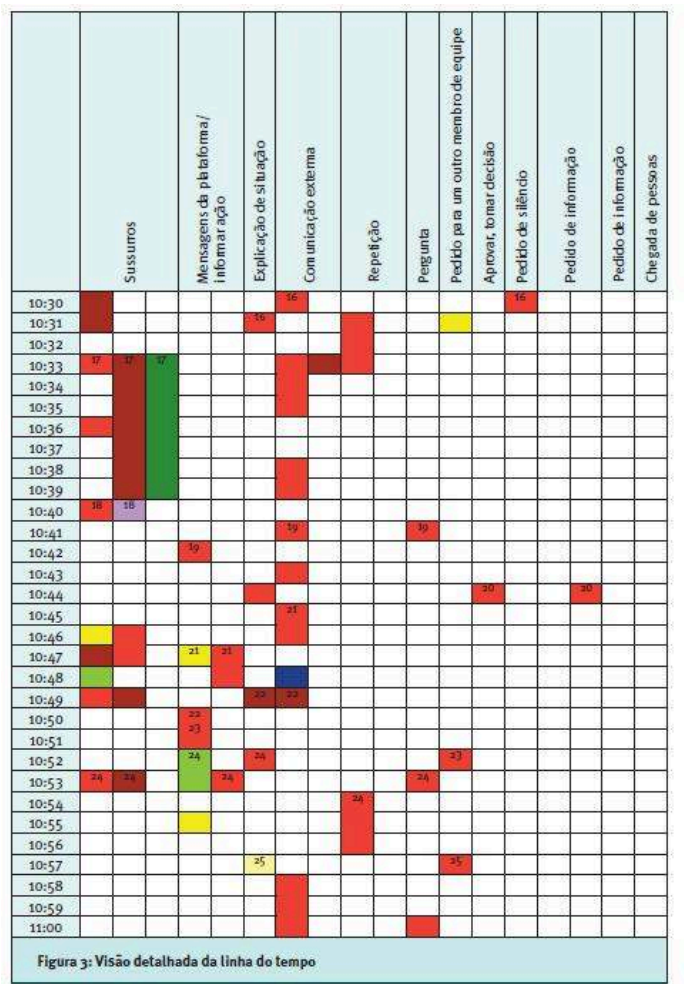

O estudo permitiu identificar fatores de resiliência e fragilidade no processo de reposta à emergência nuclear. Resiliência é definida como a capacidade do sistema em lidar com distúrbios, incluindo surpresas, com sucesso. As seguintes fontes de resiliência foram identificadas na análise combinada dos dados :

1. Há grandes esforços do coordenador da equipe de emergência na passagem de instruções e na manutenção de um ambiente comum a todos. Devido às características dinâmicas de uma resposta de emergência, revisões e instruções ad hoc são extremamente importantes. (Woods \& Hollnagel, 2006).

2. A diversidade na equipe de resposta às emergências pode ser uma fonte de resiliência. A presença de representantes de 26 diferentes agências revela a diversidade do grupo. Todavia, Hong e Page (2004) consideram que, grupos especializados na resolução de problemas com integrantes dotados de habilidades individuais podem superar um time de especialistas.

3. A equipe de resposta às emergências apresenta alguns bons padrões de organização. Klein (2001) define organização como a tentativa por parte de múltiplas entidades em atuar juntas com o intuito de alcançar um objetivo comum, através da realização de um plano compreendido por todos. No estudo da PEE, os membros que compõem a equipe trazem seus próprios planos e roteiros para a resposta à emergência. Para demandas mais complexas, faz mais sentido ter planos modulares ao invés de planos completos e complexos (idem).

4. Há, também, um mecanismo de reorganização que decorre das atividades da equipe de resposta às emergências. Quando surge um incidente que demanda de competências distintas, os membros são requisitados a avaliar a situação e a tomar decisões em domínios específicos, se reunindo em pequenos grupos para discutir sobre o tema. Um exemplo disso foi um caso ocorrido numa situação real não planejada no Protocolo de Simulação. Ativistas ambientais estavam bloqueando estradas perto da área da Central de Energia Nuclear. Para solucionar o problema, representantes das polícias (rodoviária, investigativa e militar) se juntaram para discutir sobre o problema e tomar decisões. Após isso, cada um deles contatou 
suas agências para agir sob coordenação e os ativistas foram controlados poucos minutos depois. Klein (2001) classifica esses mecanismos de organização como novas fontes de valor marginal para as operações.

37 A Fragilidade de um sistema são aspectos que tornam o funcionamento do sistema mais perigoso (com mais chances de produzir saídas inadequadas). Identificar fontes de fragilidade pode ajudar a antecipar como o sistema pode falhar, auxiliando a prevenção (Gomes, Woods, Carvalho \& Borges, 2009).

\section{As fontes de fragilidade identificadas foram :}

1. Enquanto um acidente nuclear é extremamente complexo e dinâmico, a concepção da atual simulação estudada foi bastante estática. Existe um número finito de eventos prédeterminados que foram enviados para a equipe de resposta às emergências em uma seqüência também pré-determinada, criando um cenário menos complexo e desafiador. Houve uma notável mudança no comportamento na equipe quando uma situação real e inesperada ocorreu e um grupo de ativistas começou a bloquear as estradas. Os participantes da agência ficaram visivelmente mais sérios e as atividades marginais entre as pessoas que não estavam concentradas em encontrar soluções para o problema diminuíram.

2. Por mais que existam mecanismos de passagem de instruções repetidamente durante toda a simulação, não há mecanismos específicos capazes de transmitir um parecer da situação aos agentes que chegam durante o evento ou durante o processo de tomada de decisão. A chegada dessas pessoas ocorre, geralmente, nas primeiras horas da simulação quando as agências contratadas estão enviando seus representantes, durante a hora do almoço ou, também, durante o curso da simulação, quando a substituição de um agente é necessária por algum motivo.

3. A distribuição física dos indivíduos nas salas é extremamente importante, uma vez que há situações em que combinações diferentes de agências vão interferir mais do que outras. Em ambientes de trabalho compartilhados, os indivíduos irão organizar seus lugares e atividades de acordo com a distribuição dos outros na sala. (Engeström \& Middleton, 1996). Uma organização com postos de trabalho apropriados e flexíveis é capaz de promover um layout dinâmico das células representativas das agências, aperfeiçoando o existente mecanismo de reposta à emergência.

4. As atividades realizadas pelo coordenador da equipe de simulação são extremamente importantes para a execução e comando da PEE. No entanto, se a maior parte das atividades da PEE é realizada pelo coordenador, pode haver uma sobrecarga cognitiva, gerando, desta forma, um gargalo no processo de tomada de decisões. Grande parte das atividades de comunicação entre os agentes é feita pelo coordenador, podendo representar uma sobrecarga nas suas atividades.

5. O número de agentes e de agências tem influência na organização e no desempenho da equipe. Após algumas horas na sala da emergência externa, parece ocorrer uma tendência de dispersão dos participantes e, provavelmente, uma perda de concentração. Tal fato é comprovado pela quantidade de vezes em que é pedido silêncio à equipe. O número de indivíduos pode degradar esse contexto, especialmente se alguns dos agentes não têm participação ativa nas decisões e nas atuações da equipe. Ter uma equipe é melhor apenas quando o desempenho do grupo é maior do que somatório individual do de cada membro. Quanto mais integrantes, maior o custo de coordenação e a equipe pode se tornar excessivamente numerosa.

6. Embora as agências tragam os seus próprios planos de emergência, gerando um plano de emergência modular menos complexo, é necessário um plano elaborado para identificar a função e o papel de cada agência em resposta à emergência nuclear. Uma análise do PEE atual pode aperfeiçoar a resiliência de simulação. 
7. Há uma deficiência na estrutura tecnológica visual e de comunicação utilizada por todos os agentes envolvidos no Plano de Emergência para entender e compartilhar a situação de emergência. Todas as descrições dos eventos e das atividades são feitas verbalmente. A estrutura tecnológica visual e de comunicação é importante para entender o contexto e para a tomada de decisão quando o tempo de resposta é curto (Schoenwald, Trent, Tittle \& Woods, 2005).

\section{Conclusões}

Nos estudos de caso aqui apresentados, foram utilizadas múltiplos métodos e técnicas da ATC para encontrar fontes de resiliência e de fragilidade nos domínios da produção de energia nuclear, na aviação offshore de helicópteros na Bacia de Campos, bem como na simulação da resposta à emergência nuclear. Na nossa análise encontramos essas fontes ligadas à coordenação de equipe, à concepção e à dinâmica do projeto das organizações e das relações inter-organizações, do design da simulação em si e dos cenários, ao design das estações de trabalho, à estrutura tecnológica visual e de comunicação e às atividades de resposta à crise.

Conhecer e compreender estas fontes no sistema é um mecanismo útil para melhor compreender o porquê do sucesso ou do fracasso das atividades e a interferência do sistema no desempenho.

No acidente da nave Colúmbia, o sistema de gerenciamento de segurança da NASA falhou em entender as implicações na segurança do tradeoff realizado pelas pessoas, relacionado ao vazamento de espuma que ocorreu em quase todos os vôos. Uma situação similar ocorreu no acidente Challenger relacionado às condições dos O-rings. A falta de compreensão da imagem global, no que diz respeito ao vazamento de espuma durante os lançamentos anteriores que contribuíram para os tradeoffs antes e durante a última missão do ônibus espacial Colúmbia (e.g. Por que o vídeo do vazamento de espuma durante o lançamento foi ignorado pelo comando da missão?), está diretamente ligado às decisões locais tomadas pelas pessoas dentro dos vários níveis da organização.

2 Novos estudos e pesquisas se fazem necessários para avançar o conhecimento nestes três domínios, com o objetivo de analisar profundamente essas fontes de resiliência, além de reduzir as fontes de fragilidades ou até transformá-las em fontes de resiliência. Os resultados fornecidos por essa análise sugerem que áreas como coordenação de equipe, concepção e dinâmica da simulação, gestão de crises e o desenvolvimento da estrutura tecnológica necessária para suporte são áreas com elevado potencial para aperfeiçoamento na simulação de resposta à emergência.

3 Portanto, um sistema proativo de gerenciamento de segurança usando conceitos da engenharia de resiliência deve fornecer à organização meios eficazes para balancear segurança e objetivos de alta produtividade, através da reestruturação das interações entre níveis para melhor balancear segurança com pressões de produção. Para isso, segurança precisa ser tratada como algo fundamental, monitorando continuamente o modelo de risco e decisões de sacrifício que as pessoas usam em suas atividades diárias.

Essas organizações resilientes devem fugir do viés da falta de prevenção, situação na qual temos que esperar por acidentes para pensar em segurança. De fato, como indicado por Weick "safety is a dynamic non event", (Weick, 1993), ou em outras palavras, o nível de segurança vigente permanece despercebido se não acontecer nada. 
Ao invés de ver o sucesso passado como um indicador de um bom nível de segurança, organizações resilientes devem continuar a investir em antecipar mudanças para prevenir falhas potencias, compreendendo sempre que seu conhecimento é imperfeito e que seu ambiente muda constantemente.

\section{BIBLIOGRAFIA}

Adamski, A. \& Westrum, R. (2003). Requisite imagination. The fine art of anticipating what might go wrong. In E. Hollnagel (Ed.), Handbook of cognitive task design (pp. 193-220). Mahwah, NJ : Lawrence Erlbaum Associates.

CAIB (2003). Columbia Accident Investigation Board. Report, 6 vols. Washington DC : Government Printing Office.

Carvalho, P., Santos, I., Gomes, J. \& Borges, M. (2008). Micro incident analysis framework to assess safety and resilience in the operation of safe critical systems : a case study in a nuclear power plant. Journal of Loss Prevention in the Process Industries, 21/3, 277-286.

Carvalho, P., Gomes, J., Huber, G. \& Vidal, M. (2009). Normal people working in normal organizations with normal equipment : system safety and cognition in a mid-air collision. Applied Ergonomics, 40, 325-340.

Carvalho, P., Santos, I. \& Vidal, M. (2006). Safety implications of cultural and cognitive issues in nuclear power plant operation. Applied Ergonomics, 37, 211-223.

Carvalho, P., Vidal, M. \& Carvalho, E. (2007). Nuclear power plant communications in normative and actual practice : A field study of control room operators' communications. Human Factors in Ergonomics and Manufacturing, 17, 43-78.

Cook, R., Render, M. \& Woods, D. (2000). Gaps in the continuity of care and progress on patient safety. British Medical Journal, 320, 791-794. Costa et al., (2008). Resilience and Brittleness in a Nuclear Emergency Response Simulation : Focusing on Team Coordination Activity. In Prodeedings of the 3rd Resilience Engineering Symposium, Juanles-Pins, France.

Crandall, B., Klein, G.\& Hoffman, R. (2006). Working Minds : a Practitioners Guide to Cognitive Task Analysis. Cambridge : MIT Press.

Dekker, S. (2006). Resilience Engineering : Chronicling the Emergence of Confused Consensus. In E. Hollnagel, D.D. Woods and N. Leveson (Eds.), Resilience Engineering : Concepts and Precepts. Aldershot, UK : Ashgate.

Engeström, Y.\& Middleton, D.(Eds.)(1996). Cognitionandcommunication at work. Cambridge, UK : Cambridge University Press.

Gomes, J., Woods, D., Carvalho, P. \& Borges, M.(2009). Resilience and brittleness in the offshore helicopter transportation system : The identification of constraints and sacrifice decisions in pilots' work. Reliability Engineering and System Safety, 94, 311-319.

Hollnagel, E. \& Woods, D. (2005). Joint Cognitive Systems : An Introduction to Cognitive Systems Engineering. Oxford : Taylor \& Francis. 
Hong, L. \& Page, S. (2004). Groups of diverse problem solvers can outperform groups of highability problem solvers. New York : New York University.

Klein, G. (2001). Features of team Coordination. In M. McNeese, E. Salas, M. Endsley (Eds.), New Trends in Cooperative Activities : System Dynamics in Complex Environments. Santa Monica, CA : Human Factors and Ergonomics Society Press.

Perin, C. (2005). Shouldering risks : the culture of control in the nuclear power industry. New Jersey : Princeton University Press.

Schoenwald, J., Trent, S., Tittle, J. \& Woods, D. (2005). Scenarios as a tool for design envisioning : Using the case of new sensor technologies for military urban operations. Columbus : Ohio State University.

Snook, S. (2000). Friendly Fire : The Accidental Shootdown of US Black Hawks Over Northern Irak. New Jersey : Princeton University Press. Weick, K. (1993). The Collapse of Sense Making in Organizations : the Mann Gulch Disaster. Administrative Science Quarterly, 38, (4), 628-652.

Woods, D. (2005). Creating Foresight : Lessons for Resilience from Columbia. In W. H. Starbuck and M. Farjoun (Eds.), Organization at the Limit : NASA and the Columbia Disaster. Malden, MA : Blackwell.

Woods, D. (2006). Essential Characteristics of Resilience for Organizations. In E. Hollnagel, D. Woods and N. Leveson (Eds.), Resilience Engineering : Concepts and Precepts. Aldershot, UK : Ashgate.

Woods, D. \& Hollnagel, E. (2006). Joint Cognitive Systems : Patterns in Cognitive Systems Engineering. Boca Raton, FL : Taylor and Francis.

\section{RESUMOS}

A Engenharia de Resiliência tem como propósito tornar pessoas e organizações atentas e sensíveis aos modelos de riscos adotados e às estratégias adaptativas usadas para controlar a emergência e os percursos das falhas. Uma organização resiliente deve proporcionar meios para a gestão das adaptações, monitorando, entendendo, refletindo e aprendendo a partir dessas estratégias, identificando ameaças e riscos à segurança. Falhar em aplicar esses princípios leva a organização a atuar em um modo reativo, numa condição de luta constante contra o perigo. Os estudos de caso apresentados neste artigo procuram aplicar os conceitos e métodos de engenharia resiliência, bem como relacioná-los com o conceito de sustentabilidade dos sistemas sócio-técnicos complexos, num país de desenvolvimento industrial recente como é o caso do Brasil. Os casos abordam os domínios nuclear, aviação e emergência e utilizam a Análise do Trabalho Cognitivo (ATC) como base metodológica. Os estudos permitiram identificar fatores de resiliência e fragilidade nos diversos domínios abordados, mostrando ainda que um sistema proativo de gerenciamento de segurança, usando conceitos da engenharia de resiliência, pode fornecer às organizações meios eficazes para balancear segurança e objetivos de alta produtividade.

La Ingeniería de Resiliencia hay como propósito permitir a las personas y las organizaciones a se tornaren atentas y sensibles a los modelos de riesgos adoptados y a las estrategias adaptativas usadas para controlar la emergencia y los recorridos de fallas. Una organización resiliente debe proporcionar medios para la gestión de las adaptaciones, monitoreando, entendiendo, reflejando y aprendiendo a partir de estas estrategias, identificando amenazas y riesgos à la seguridad. Fallar en aplicar eses principios lleva la organización a actuar en un modo reactivo, en una 
condición de lucha constante contra el peligro. Los estudios de caso presentados en este artigo procuran aplicar los conceptos y métodos de la ingeniaría de resiliencia, así como relaciónalos con el concepto de sustentabilidad de los sistemas socio-técnicos complejos, en un país de desarrollo industrial reciente que es el caso de Brasil. Los casos abordan los dominios nuclear, aviación y emergencia y utilizan la Análisis del Trabajo Cognitivo (ATC) como base metodológica. Los estudios permitirán identificar factores de resiliencia y fragilidad en los diversos dominios abordados, mostrando aun que un sistema proactivo de gerenciamiento de seguridad usando conceptos de la ingeniería de resiliencia puede fornecer a las organizaciones medios eficaces para balancear seguridad y objetivos de alta productividad.

Les concepts et le cadre théorique de l'ingénierie de la résilience visent à sensibiliser les individus et les organisations aux modèles du risque adoptés, ainsi qu'aux stratégies d'adaptation choisies, pour contrôler l'émergence de défaillances ainsi que les voies par lesquelles elles surviennent. Une organisation résiliente doit donner les moyens aux acteurs du système de gérer ces adaptations par la surveillance, la compréhension, la réflexion, et l'apprentissage à partir de ces stratégies, identifiant les menaces et les risques existants en termes de sécurité. Ne pas appliquer ces principes pousse l'organisation à agir suivant un mode réactif, à lutter constamment contre le danger. Les études de cas présentées dans cet article cherchent à appliquer les concepts et méthodes de l'ingénierie de la résilience, mais aussi à les rattacher au concept du développement durable des systèmes sociotechniques complexes dans un pays d'industrialisation récente tel que le Brésil. Ces études de cas proviennent des domaines de l'industrie nucléaire et de l'aéronautique et s'appuient sur le cadre méthodologique de l'analyse des activités cognitives. Ces études ont permis d'identifier des facteurs de résilience et de fragilité dans ces divers domaines, et soulignent qu'un système proactif de gestion de la sécurité fondé sur les concepts de l'ingénierie de la résilience peut fournir des moyens efficaces pour équilibrer les objectifs de sécurité et de productivité.

Resilience Engineering aims to make people and organizations sensitive to the risk models adopted and to the adaptive strategies used to control the emergency and the failure paths. A resilient organization must provide means to the management of adaptations by monitoring, understanding, reflecting and learning from these strategies, and by identifying threats and risks to safety. The failure in applying such principles leads the organization to act in a reactive model, in an endless fight against danger. The case studies presented in this article seek to apply the concepts and methods of resilience engineering, relating them to the concept of complex sociotechnical systems' sustainability, in a recently industrialized country like Brazil. The cases approach the domains of nuclear, aviation, and emergency, using Cognitive Task Analysis as methodological basis. The studies have permitted to identify resilience and brittleness factors in the several domains researched; pointing out that a proactive safety management system based on resilience engineering concepts may provide the organizations with effective means to balance safety and productivity goals.

\section{ÍNDICE}

Mots-clés: résilience, sûreté de systèmes complexes, analyse du travail cognitif

Palabras claves: resiliencia, seguridad de sistemas complejos, análisis del trabajo cognitivo

Palavras-chave: resiliência, segurança de sistemas complexos, análise do trabalho cognitivo

Keywords: resilience, safety of complex systems, cognitive work analysis 


\section{AUTORES}

\section{JOSÉ O. GOMES}

Núcleo de Computação Electrónica \& Instituto de Matemática Universidade Federal do Rio de Janeiro, Av. Athos da Silveira Ramos, 274 Cidade Universitária, Rio de Janeiro, Brasil joseorlando@nce.ufrj.br

\section{PAULO V. R. CARVALHO}

Núcleo de Computação Electrónica \& Instituto de Matemática Universidade Federal do Rio de Janeiro, Av. Athos da Silveira Ramos, 274 Cidade Universitária, Rio de Janeiro, Brasil. Comissão Nacional de Energia Nuclear Instituto de Engenharia Nuclear, Brasil 2 paulov@ien.gov.br

\section{DAVID D. WOODS}

Institute for Ergonomics Ohio State Universit, EUA

woods.2@osu.edu

\section{TAHAR HAKIM BENCHEKROUN}

Laboratoire d'Ergonomie, Conservatoire National des Arts et Métiers, France tahar-hakim.benchekroun@cnam.fr

\section{MARCOS R. S. BORGES}

Núcleo de Computação Electrónica \& Instituto de Matemática Universidade Federal do Rio de Janeiro, Av. Athos da Silveira Ramos, 274 Cidade Universitária, Rio de Janeiro, Brasil mborges@nce.ufrj.br 